BULL. AUSTRAL. MATH. SOC.

\title{
An embedding theorem for fields: Addendum
}

\section{J.W.S. Cassels}

The proof of Lemma 2 in [1] invoked elementary analytic number-theory. I have just realized that there is a proof which is entirely elementary. It is doubtless "well-known" (in the usual technical sense that it appears somewhere in the literature) and it is certainly "well-knowable" in Conway's terminology. However, as it renders the entire argument of my paper elementary, I give it here.

The lemma asserts that if

$$
f(x)=f_{n} x^{n}+f_{n-1} x^{n-1}+\ldots+f_{0}
$$

is a non-constant polynomial with rational integral coefficients, then there are infinitely many primes $p$ for which there is an integer $b$ satisfying

$$
f(b) \equiv 0(\bmod p) \text {. }
$$

If $f_{0}=0$ we can take $b=0$ for any prime $p$ : so we can suppose that

$$
f_{0} \neq 0
$$

Suppose, if possible, that (2) has a solution only for the primes $p$ in the finite set $P$ (possibly empty). Let $c$ be any integer which is divisible by all the $p \in P$. Then

$$
f\left(f_{0} c\right)=f_{0} r
$$

where

$$
r=f_{n} f_{0}^{n-1} c^{n}+f_{n-1} f_{0}^{n-2} c^{n-1}+\ldots+1
$$

Received 5 March 1976. 
is prime to $c$, and, in particular, is not divisible by any $p \in P$.

Since $f(X)$ is non-constant by hypothesis, we may certainly pick $c$ so that $r \neq \pm 1$. Let $p^{*}$ be a prime dividing $r$, so $p^{*} \neq P$. By (4) we have

$$
f\left(b^{*}\right) \equiv 0\left(\bmod p^{*}\right)
$$

with $b^{*}=f_{0} c$. This contradicts the assumption that $P$ contains all the primes $p$ for which (2) is soluble and so proves the lemma.

\section{Reference}

[1] J.W.S. Cassels, "An embedding theorem for fields", BuZZ. Austral. Math. Soc. 14 (1976), 193-198.

Department of Pure Mathematics and Mathematical Statistics, University of Cambridge,

Cambridge,

England. 